ARTICLE

https://doi.org/10.1038/s41467-021-26857-w

\title{
Photo-induced catalytic halopyridylation of alkenes
}

\author{
Shi-Yu Guo ${ }^{1}$, Fan Yang ${ }^{1}$, Ting-Ting Song ${ }^{1}$, Yu-Qing Guan ${ }^{1}$, Xiang-Ting Min ${ }^{1}$, Ding-Wei Ji ${ }^{1}$, Yan-Cheng Hu (1) ${ }^{1} \&$ \\ Qing-An Chen (1) 1,2凶
}

The Mizoroki-Heck reaction and its reductive analogue are staples of organic synthesis, but the ensuing products often lack a chemical handle for further transformation. Here we report an atom-economical cross-coupling of halopyridines and unactivated alkenes under photoredox catalysis to afford a series of alkene halopyridylation products. This protocol with mild and redox neutral conditions contributes broad substrate scope. As a complement to conventional Heck-type reaction, this radical process avoids the involvement of $\beta$ - $\mathrm{H}$ elimination and thus useful pyridyl and halide groups could be simultaneously and regioselectively incorporated onto alkenes. The success depends on TFA-promoted domino photocatalytic oxidative quenching activation and radical-polar crossover pathway. Plausible mechanism is proposed based on mechanistic investigations. Moreover, the reserved $\mathrm{C}-\mathrm{X}$ bonds of these products are beneficial for performing further synthetic elaborations.

\footnotetext{
${ }^{1}$ Dalian Institute of Chemical Physics, Chinese Academy of Sciences, 457 Zhongshan Road, 116023 Dalian, China. ${ }^{2}$ University of Chinese Academy of Sciences, 100049 Beijing, China. ${ }_{\text {email: qachen@dicp.ac.cn }}$
} 
T he rapid elaboration of molecular complexity from simple substrates has always been a fundamental theme in organic chemistry. Among those known methods, catalytic functionalizations of alkenes have gained much attention owing to the low cost and easy availability ${ }^{1-9}$. Of particular note is classical Heck cross-coupling that provides a rapid access to arylated alkenes (Fig. 1a) ${ }^{10-14}$. Besides, with an introduction of external hydride sources, a variety of reductive Heck-type products could be readily obtained via hydroarylation of alkenes (Fig. 1b) ${ }^{15-18}$. While they have wide applicability, both processes involve an elimination of halide atoms as inevitable waste.

On the other hand, the selective construction of $\mathrm{C}-\mathrm{X}$ bond is of great significance as it provides an important synthetic handle for the incorporation of diverse functional groups ${ }^{19-25}$. In this context, it is appealing to exploit a complementary cross-coupling featuring installation of new $\mathrm{C}-\mathrm{C}$ and $\mathrm{C}-\mathrm{X}$ bonds onto alkenes simultaneously without sacrifice of any atoms. Following this concept, elegant intramolecular haloarylations of alkenes have been established by Lautens, Glorius, Tong etc ${ }^{26-35}$. Their success usually relies on the employment of terminal disubstituted alkenes that can lead to an alkyl Pd or Ni halide intermediate without a syn $\beta$-H atom (Fig. 1c). Besides, the haloarylation of ethylene and propylene with aryl chlorides normally requires carcinogenic high-energy ultraviolet (UV) light ${ }^{36}$. Despite these impressive advances, visible light-induced catalytic intermolecular haloarylation of wide simple alkenes with aryl halides still remains underexplored.

In recent years, photoredox catalysis ${ }^{37-43}$ represents the state-ofthe-art technique to promote functionalizations of alkenes ${ }^{44-48}$. Owing to the ubiquitous role of pyridyl motifs as aromatic heterocycle in ligand scaffolds, natural products, and medically relevant molecules ${ }^{49-53}$, significant efforts have been devoted to visible lightdriven pyridylation of alkenes ${ }^{54-63}$. Intrigued by these precedents and our continued interest on alkenes functionalizations ${ }^{64-67}$, we questioned whether it is possible to simultaneously install both pyridyl and halide groups onto alkenes which possess a potential syn $\beta$ - $\mathrm{H}$ atom.

Herein, this goal has been achieved by coupling of unactivated alkenes and halopyridines under visible light condition (Fig. 1d). This halopyridylation transformation is enabled by domino photocatalytic oxidative quenching activation and radical-polar crossover pathway. It provides an important complement for known precedents about Heck reactions with respect to product diversity and atom economy.

\section{Results}

Reaction optimization. Initially, under blue light irradiation, 2-bromo-6-methylpyridine 1a and 1-hexene 2a were selected as

a) Classical Heck reaction: Catalytic arylation of alkenes

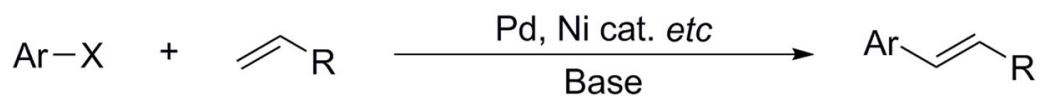

b) Reductive Heck reaction: Catalytic hydroarylation of alkenes

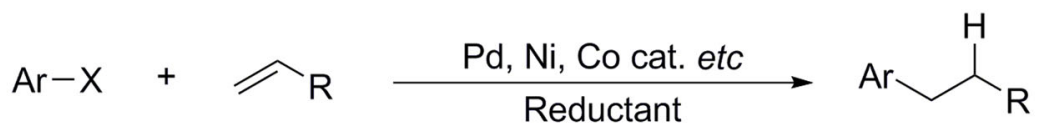

c) Intramolecular haloarylation of alkenes (without syn $\beta$-H)
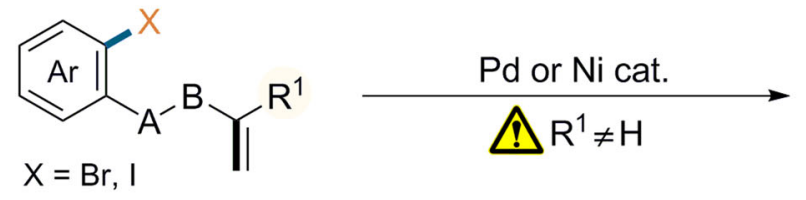<smiles>[R]C1(CI)[B][Te]c2ccccc21</smiles>

Contributed by Lautens, Glorius, Tong, Qiu, Zhang, Li etc.

d) This work: Photo-induced catalytic halopyridylation of alkenes

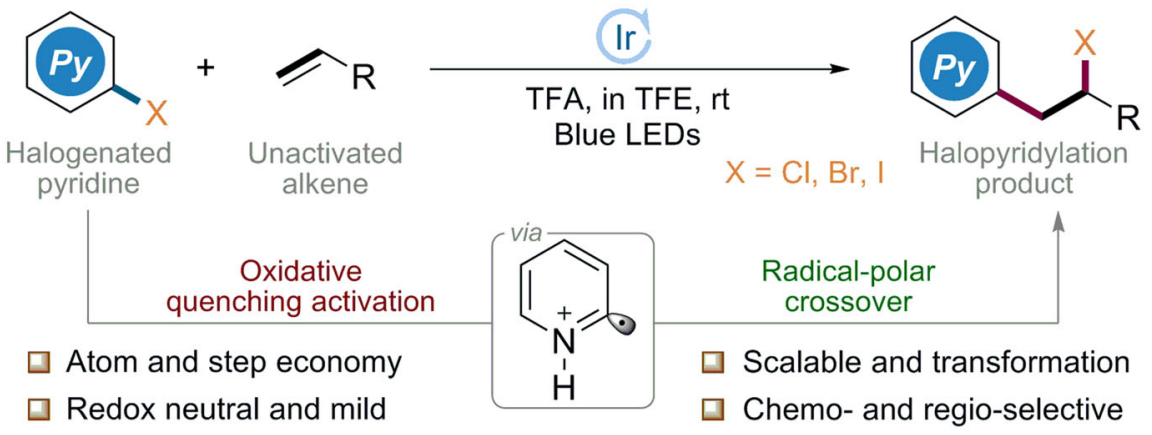

Fig. 1 Cross-coupling of aryl halides with alkenes. a Classical Heck reaction. b Reductive Heck reaction. c Intramolecular halo alytic halopyridylation of alkenes. d Photo-induced catalytic halopyridylation of alkenes. 
model substrates to optimize the reaction (Table 1 and Supplementary Tables 1-8 in Supplementary Information). First, as state-of-the-art photocatalytic strategy of pyridyl radical generation, Jui's elegant protocols ${ }^{56,59}$ were applied to these substrates. Both results clearly shown that there was no detectable bromopyridylation product 3aa formed under Jui's conditions (Table 1, entries 1-2). Only a trace amount of product 3 aa could be detected in the absence of HEH (Hantzsch ester) (entries 3-4). Based on these results, Jui's reductive quenching activation of halopyridine conditions were not suitable for the desired C-X bond formation. According to general principle for the construction of $\mathrm{C}-\mathrm{X}$ bond, we envisioned that oxidative quenching activation of halopyridine would divert the reactivity from hydropyridylation towards halopyridylation of alkenes.

After careful evaluation for oxidative quenching activation of bromopyridine 1a, the desired bromopyridylation product 3aa was furnished in $89 \%$ yield using $\left[\operatorname{Ir}(\mathrm{ppy})_{2}(\mathrm{dtbbpy})\right] \mathrm{PF}_{6}(\mathrm{Ir}-\mathrm{PC}$ A) as photocatalyst and TFA (Trifluoroacetic acid) as additive (Table 1, entry 5). No target product was obtained in the absence of photocatalyst or in dark environment (entries 6 and 7). Strong electron-deficient photocatalyst (Ir-PC B) and tris(2-phenylpyridine)iridium (Ir-PC D) showed no catalytic performance, while 4-fluorophenylpyridine derived catalyst (Ir-PC C) gave a slightly decreased yield (entry 8 ). Other commonly used organophotocatalysts such as Eosin $\mathrm{Y}$ and (Acr-Mes) $\mathrm{ClO}_{4}$ were also examined but found to be ineffective (entry 9). Moreover, the Brønsted acid additive was essential for the process and the acidity also exerted an important influence on the outcomes (entries 10 and 11). For instance, weak acid $\mathrm{AcOH}$ could not promote the reaction, whereas $(\mathrm{PhO})_{2} \mathrm{POOH}$ and $\mathrm{PhSO}_{3} \mathrm{H}$ delivered the target product 3aa in $76 \%$ and $80 \%$ yields, respectively (entry 11 ). Strongly polar protic solvent, such as TFE (Trifluoroethanol) and hexafluoroisopropanol (HFIP) were both competent solvents for this reaction, but EtOH was not feasible (entry 12). The reaction could not take place under thermal conditions or air atmosphere (entries 13 and 14).

Substrate scope. Having identified optimized conditions, the substrate scope with respect to alkenes was examined (Fig. 2a). To our delight, a number of terminal alkenes, including terminal $\alpha$ alkenes (3aa-3ae) and 1,1-disubstituted alkenes (3af, 3ag) could be successfully bromopyridylated under this protocol. Notably, alkenes with free hydroxyl group were suitable coupling partners as well, affording desired products in moderate to good yields (3ac, 3ad, and 3ag). In addition to terminal alkenes, this protocol could be extended to internal alkenes (3ah-3al). For example, bromopyridylation of trans-4-octene delivered the expected product 3 ah in $84 \%$ yield with 2:1 dr. A variety of cyclic internal alkenes, including cyclopentene, cyclohexene, and norbornene were also well tolerated to furnish the corresponding products in $81-90 \%$ yields (3ai-3ak). Gratifyingly, the treatment of trisubstituted alkene with standard conditions resulted in the generation of single product 3 al in $69 \%$ yield. The exclusive regioselectivity is presumably ascribed to the favorable pyridyl radical addition at the less hindered alkene carbon, which could also lead to a more stable tertiary carbon radical or carbocation. Unsurprisingly, only trace amount of bromopyridylated product 3am was observed for the coupling with tetrasubstituted alkene. When non-conjugated dienes were subjected to this reaction conditions, one carbon-carbon double bond could be selectively reserved for potential product diversity (3an and 3ao).

Subsequently, we turned our attention to the investigation on the scope of halopyridines using 1-hexene as the model alkene. As shown in Fig. 2b, a broad range of bromopyridines, including alkyl-substituted (3ba-3fa), unsubstituted (3ga, 3ha), and disubstituted bromopyridines (3ia-3ka) proved to be smoothly accommodated to this protocol. The position of alkyl substituents (methyl and alkoxy) in bromopyridines had no great influence on the yields of products (3ba-3fa). Simple 2- and 4-bromopyridines were also amenable to the transformation, but the bromine substituent at 2-position seemed to be more reactive (3ga, $3 \mathbf{h a}$ ). Notably, in the cases of 2,3- and 2,5-dibromopyridines, the reaction selectively took place at the 2-position with the preservation of 5- or 3-bromo group for further synthetic elaboration (3ia and $\mathbf{3 j a}$ ). In comparison, 2,6-dibromopyridine could react with two molecules of 1 -hexene to produce $3 \mathbf{k a}$ in $70 \%$ yield. Moreover, this protocol could also be extended to a variety of chloro and iodopyridines (3la-3pa) which might be great challenges for traditional metal catalysis in terms of substrate activation or product overreaction. However, bromopyridine substrates bearing strong electron withdrawing groups were not suitable for this reaction. It probably resulted from the protonation of electron-deficient pyridines with TFA may not be favored, which would be detrimental to product formation (Supplementary Table 9 in Supplementary Information).

To magnify the potential merit of current photocatalytic protocol, we assessed the late-stage halopyridylation of several complex scaffolds based on natural products and drug molecules, as illustrated in Fig. 2c. Specifically, terpenes substrate perilla aldehyde was feasibly transformed into the corresponding bromopyridylation product $\mathbf{4 a}$ in $75 \%$ yield and the unique chemoselectivity for terminal alkene over internal alkene was displayed. In addition, complex molecules containing diverse structural features, such as carbohydrate $(\mathbf{4 b})$, sulfonamide $(\mathbf{4 c})$, and steroid (4d) under the standard conditions were also tested to effectively deliver corresponding products in $67-85 \%$ yields, indicating that this method could give rapidly access to a wide range of valuable halide and pyridyl containing scaffolds.

Additionally, owing to the versatile role of organohalides, we further explored the reactions between different halopyridines and halogenated alkenes to synthesize a series of dihalogenated alkylpyridine compounds (Fig. 2d). To our delight, (pseudo)halosubstituted (Cl-, Br-, I-, and TsO-) alkenes could undergo halopyridylation smoothly to afford the dihalogenated alkylpyridine products $\mathbf{5 a - 5 1}$ in moderate to good yields. These results above adequately exemplify the mild nature of the photocatalytic system by bearing the high tolerance of various functional groups and demonstrate the attractive synthetic utility of this halopyridylation of unactivated alkenes method.

Mechanistic investigations. To probe the mechanism of this photo-induced alkenes halopyridylation, preliminary mechanistic investigations have been conducted (Figs. 3 and 4). The addition of radical scavengers TEMPO (2,2,6,6-Tetramethylpiperidinooxy) or BHT (Butylated Hydroxytoluene) to the reaction mixture almost inhibited this reaction (Fig. 3a).The observation of BHTpyridyl adduct 6 suggests that the pyridyl radical generation is probably involved in this transformation. Stern-Volmer fluorescence quenching experiments were performed on all reactants of 1a, 2a, TFA, and 1a+TFA mixture, respectively (Fig. 3b, c and Supplementary Figs. 3-6 in Supplementary Information). The results clearly shown that only the combination of $1 \mathbf{a}+$ TFA could dramatically quenched the excited state Ir-PC A* (Fig. 3b, c), which indicates an oxidative quenching activation and the protonated pyridine as the effective quencher in the photocatalytic quenching cycle.

In order to get insight into photoredox process, cyclic voltammetry experiments were further analyzed. As shown in 


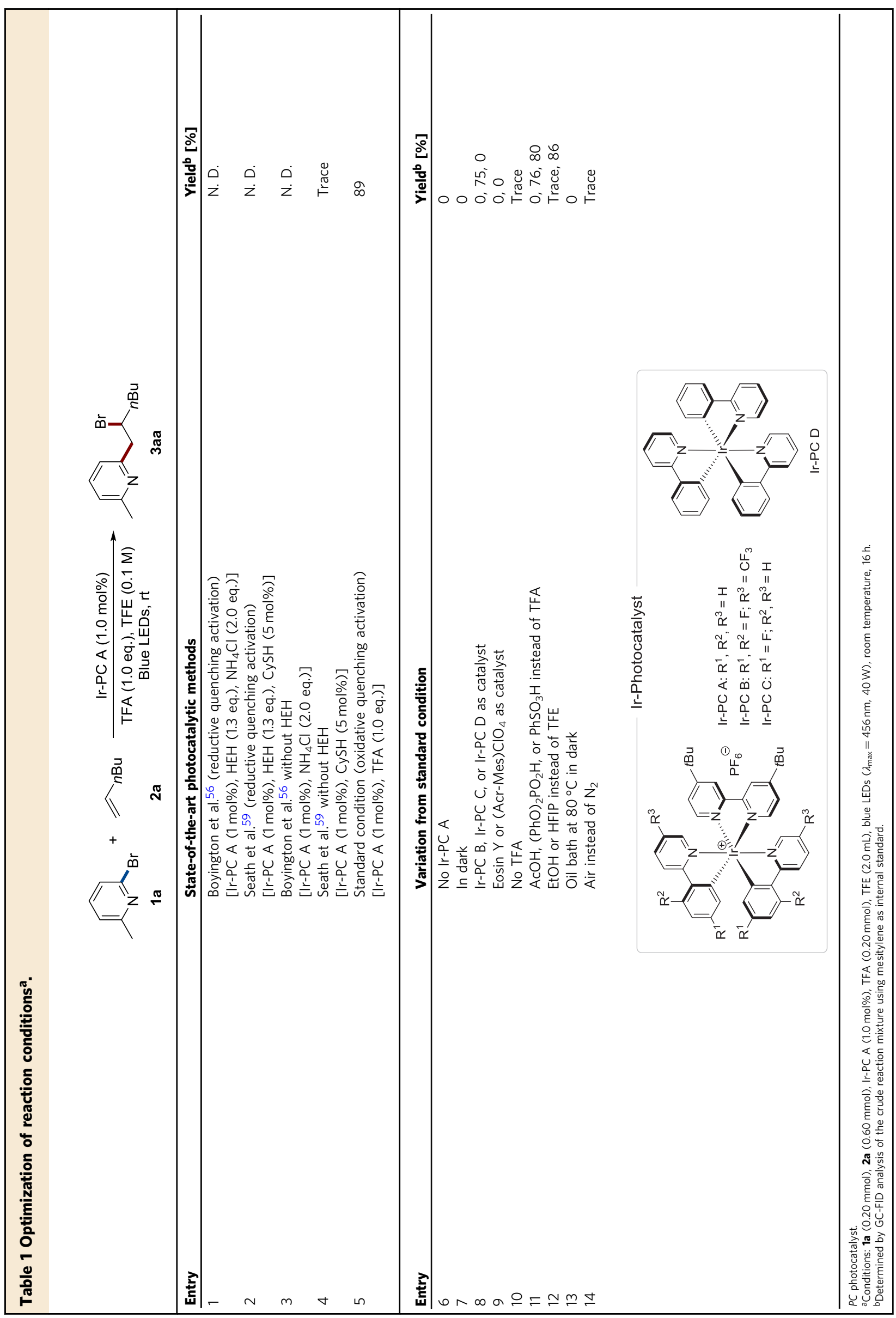



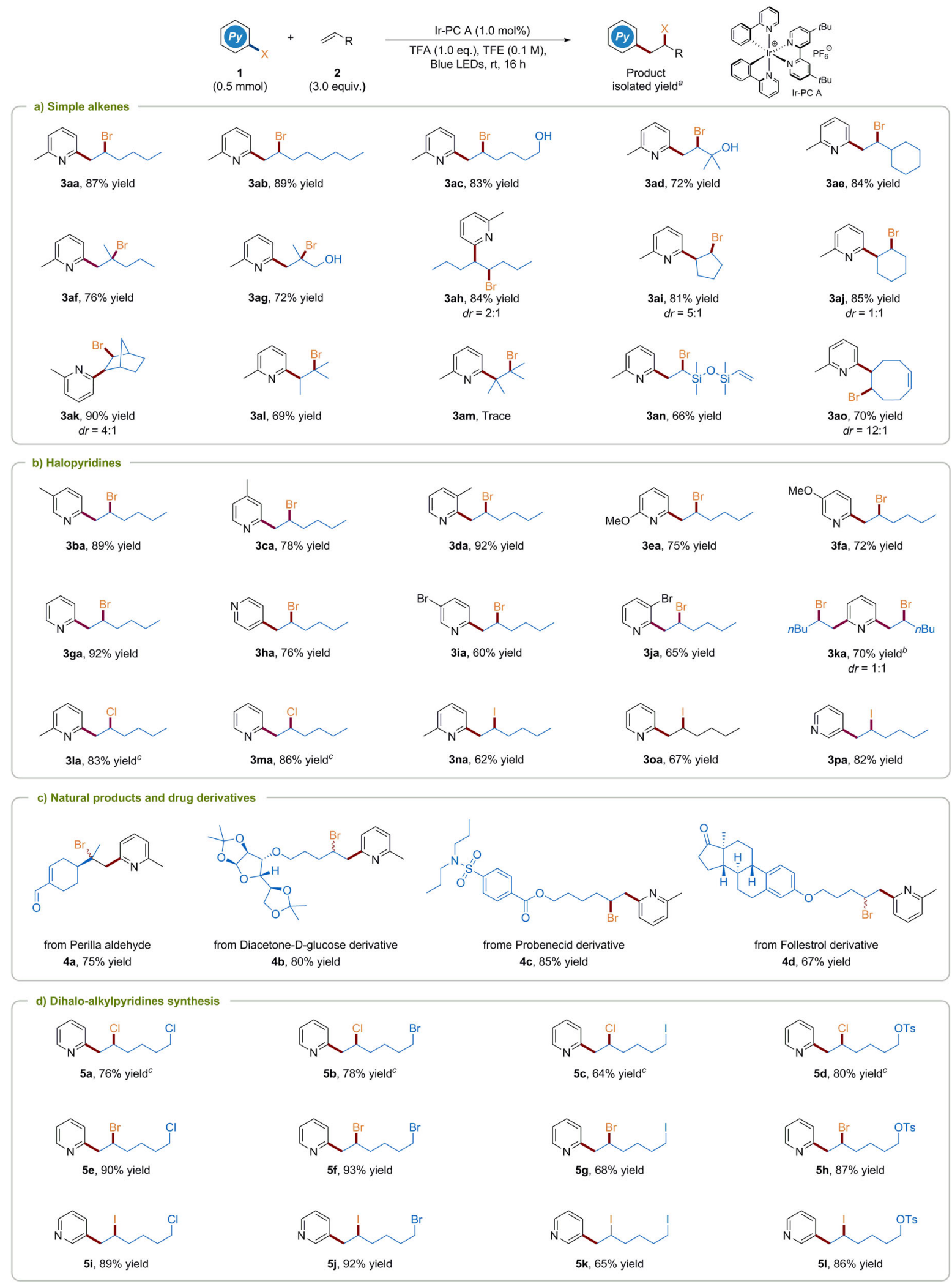

Fig. 2 Substrate scope. a Simple alkenes scope. b Halopyridines scope. c Natural products and drug derivatives. $\mathbf{d}$ Dihalo-alkylpyridines synthesis. ${ }^{\text {aYields }}$ of isolated products. Diastereoselectivity (dr) was determined by ${ }^{1} \mathrm{H}$ NMR analysis. ${ }^{6} 4.0$ equivalents of $\mathbf{2 a}$ was used. ${ }^{\mathrm{c}} 20 \mathrm{~h}$. PC photocatalyst.

Fig. 3d, a sharp reduction peak of 1 a with $E_{1 / 2}{ }^{\text {red }}=-0.76 \mathrm{~V}$ (vs. SCE) was observed in the cathodic reduction scan in TFE, and the 1a+TFA mixture (1:1 molar ratio) could rise to $E_{1 / 2}$ red $=-0.69 \mathrm{~V}$ (vs. SCE). Moreover, when the solvent was changed to EtOH, reduction potential of the $\mathbf{1 a}+\mathrm{TFA}$ mixture fell to $E_{1 / 2}$ red $=-0.95 \mathrm{~V}$ (vs. SCE) (Fig. 3e). These results suggest that the halopyridine substrate is most likely protonated first by the Brønsted acid TFA and gets stronger oxidizing properties. 
a)
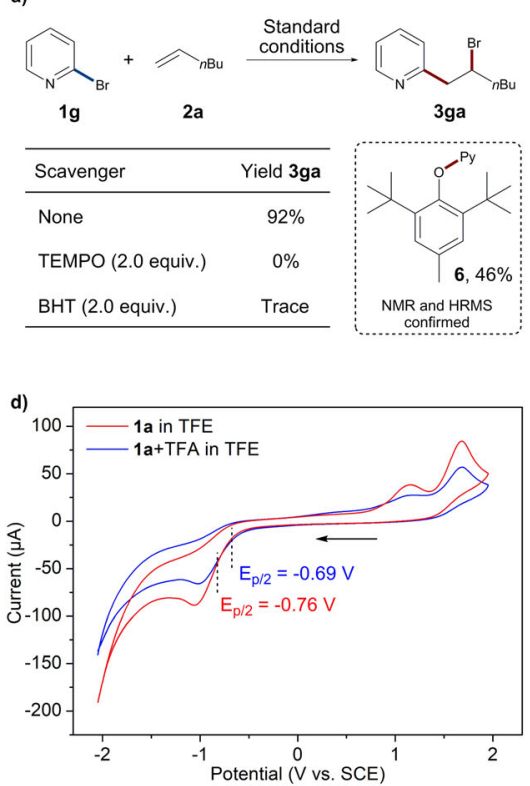

b)

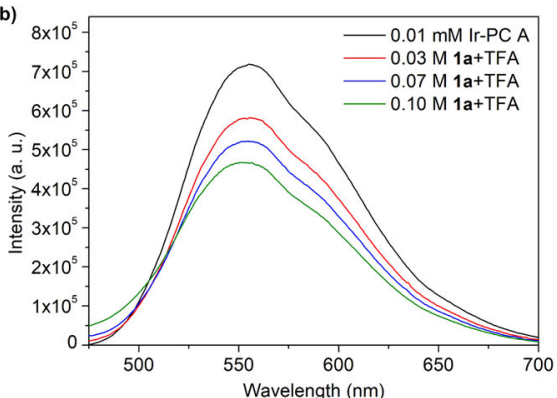

e)

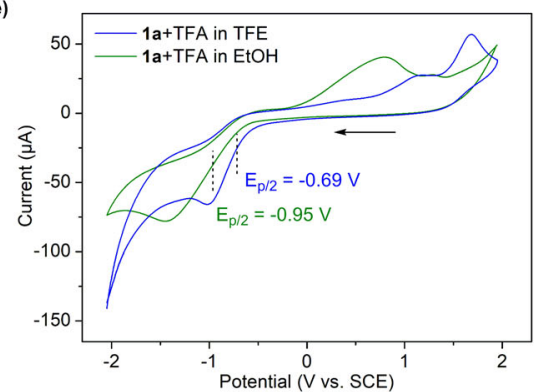

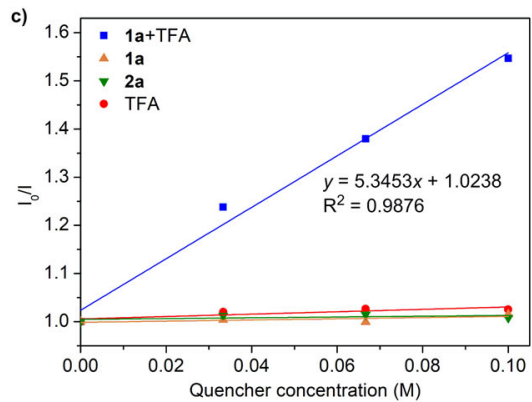

f)

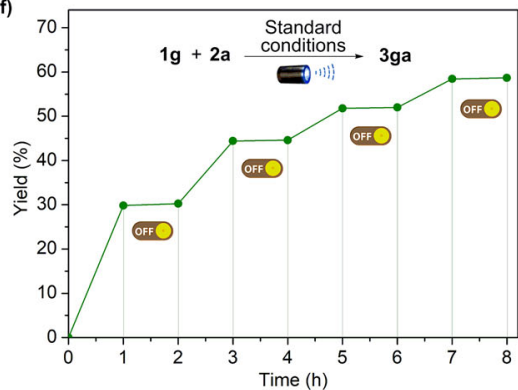

Fig. 3 Mechanistic investigations. a Radical trapping experiments. b Fluorescence quenching experiments of Ir-PC A with various concentrations of $\mathbf{1 a}+$ TFA in TFE solution. c Stern-Volmer plots of Ir-PC A with different quenchers. $\mathbf{d}$ Cyclic voltammograms of $\mathbf{1 a}$ and $\mathbf{1 a}+$ TFA in $0.1 \mathrm{M}$ TBAPF 6 TFE solution. e Cyclic voltammograms of $\mathbf{1 a}+$ TFA in $0.1 \mathrm{M} \mathrm{TBAPF}_{6}$ TFE solution and EtOH solution. $\mathbf{f}$ Light on/off experiments.

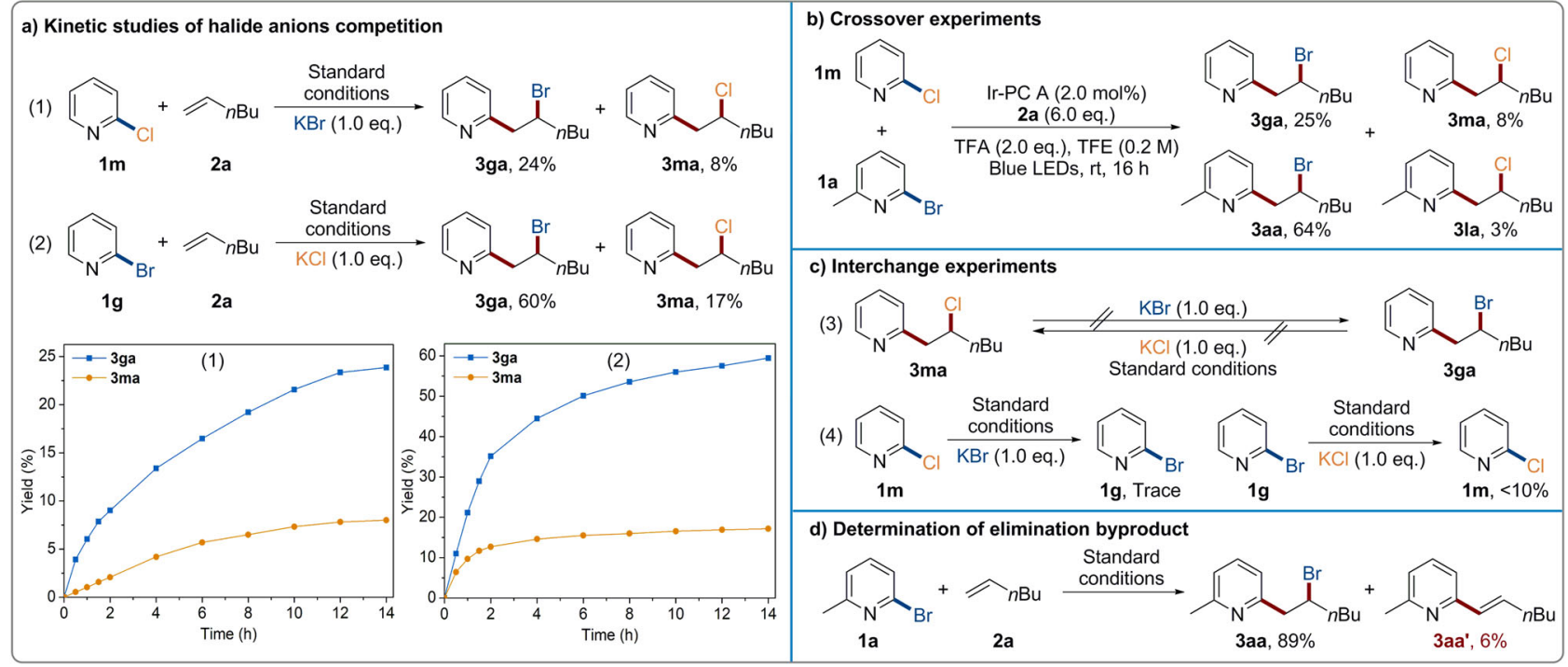

Fig. 4 Halide anions competition experiments. a Kinetic studies of halide anions competition. b Crossover experiments. c Interchange experiments. d Determination of elimination byproduct.

Light on/off control experiments were performed to obtain the reactivity profile of this protocol (Fig. $3 \mathrm{f}$ ). It is found that the product generation was blocked immediately when the light was turned off and resumed efficiently when turned on, indicating that constant light irradiation is essential for this transformation. The resultant long radical chain progress may be unlikely, considering a relatively low quantum yield $(\Phi=0.685$, Page S29 in Supplementary Information).

On the other hand, halide anions competition experiments were carried out to verify the radical-polar crossover pathway to give ATRA-type products (Fig. 4). When 2-Cl-pyridine $\mathbf{1} \mathbf{~ m}$ and 1 -hexene 2 a were subjected to this standard conditions in the presence of $\mathrm{KBr}$ (1.0 equiv.), the expected chloride product $3 \mathrm{ma}$ and crossover bromide product 3 ga were isolated in $8 \%$ and $24 \%$ yields, respectively (Fig. 4a, eq 1). Based on the kinetic studies results, the formation of crossover bromide product 3ga dominated during the whole process (Fig. 4a, chart 1). When 2Br-pyridine $\mathbf{1} \mathbf{g}$ reacted with $\mathbf{2 a}$ in the presence of $\mathrm{KCl}(1.0$ equiv.), similar but higher yields for halopyridylation products were obtained (60\% 3ga and $17 \%$ 3ma) (Fig. $4 \mathrm{a}$, eq 2$)$. With the help of these kinetic results, the higher reactivity probably resulted from the easier generation of pyridyl radical from 2-Brpyridine $\mathbf{1 g}$ than 2 -Cl-pyridine $\mathbf{1} \mathbf{~ m}$ (eq 2 vs. eq 1 ). Additionally, in the absence of extra potassium halide salt, bromide products (3ga and 3aa) also dominated over chloride derivatives (3ma and 3la) in the crossover reaction between 2-Cl-pyridine $\mathbf{1 m}$ and 2-Br-6-Me-pyridine 1a (Fig. 4b). In general, bromide anion exhibited weaker nucleophilicity than chloride one due to its weaker negative charge density. However, owing to a strong hydrogen bonding interaction in polar protic solvent TFE, the 

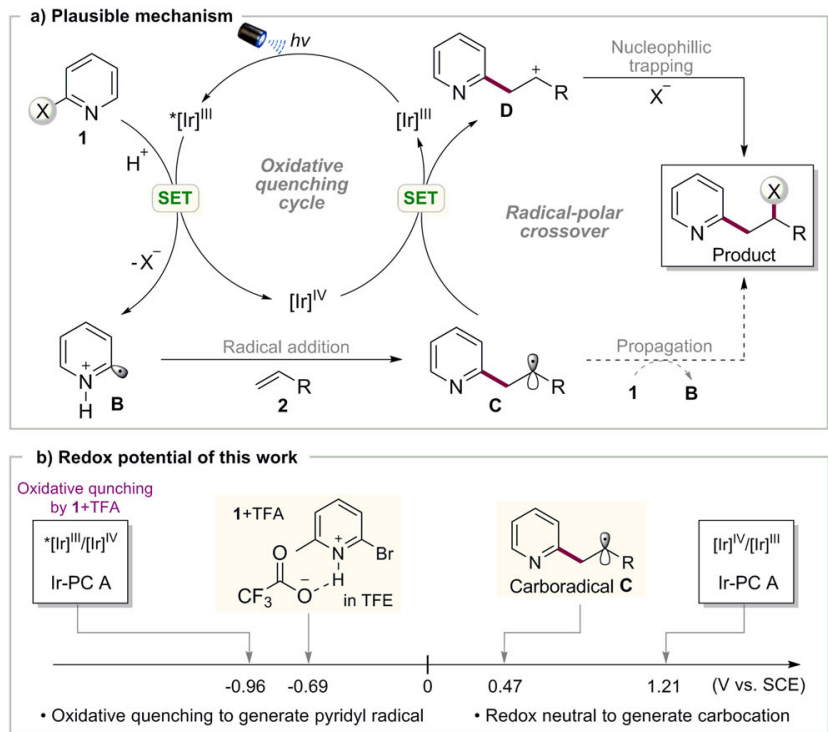
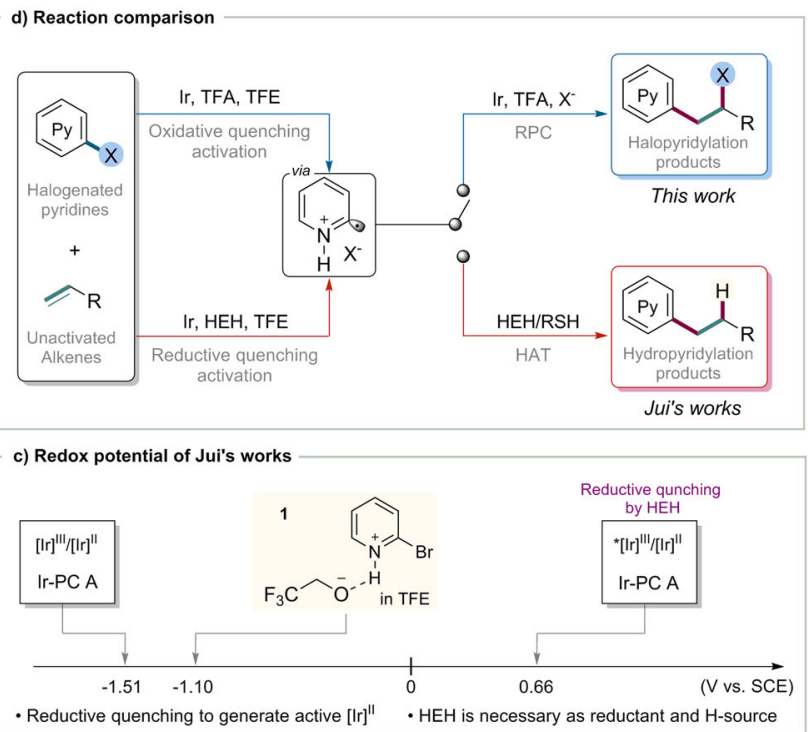

Fig. 5 Proposed mechanism and comparison. a Plausible mechanism. b Redox potential of this work. c Redox potential of Jui's works. d Reaction comparison.

nucleophilic ability was switched and bromide anion turned to be a better nucleophile. Besides, the resubjection of halide product 3ma and $3 \mathrm{ga}$ to the standard conditions delivered no detectable halide exchange products (Fig. 4c, eq 3 ). This negative halide interchange result indicates that the current photo-induced protocol will not cleavage alkyl carbon-halide bond which will be common issues for transition metal catalysis. Interestingly, the halogen exchange of halopyridines could slightly occur in the presence of extra potassium halide salt (Fig. 4c, eq 4). Moreover, Heck-type byproduct 3aa' was obtain in $6 \%$ yield under optimized reaction condition, which was formed via $\beta-\mathrm{H}$ elimination of carbon cation intermediate. This suggests that a radical-polar crossover mechanism via alkyl carbocation intermediate is operative for the C-X bond formation (Fig. $4 \mathrm{~d}$ ).

Based on the experimental results above and literatures, a possible mechanism of this photo-induced catalytic halopyridylation of alkenes is proposed in Fig. 5a. Initially, irradiation of [Ir $]^{\mathrm{II}}$ gives rise to its excited state ${ }^{*}[\mathrm{Ir}]^{\mathrm{III}}$. Then halopyridine $\mathbf{1}$ is protonated in the presence of TFA in TFE and reduced by ${ }^{*}[\mathrm{Ir}]^{\mathrm{III}}$ to generate pyridyl cation radical $\mathbf{B}$, along with $[\mathrm{Ir}]^{\mathrm{IV}}$ and halide anion. The subsequent electrophilic addition of protonated pyridyl radical $\mathbf{B}$ to nucleophilic alkene $\mathbf{2}$ produces the secondary alkyl radical C. Alkyl radical C undergoes polarity-matched SET with $[\mathrm{Ir}]^{\mathrm{IV}}$ to give carbocation $\mathrm{D}^{68,69}$, and regenerates the $[\mathrm{Ir}]^{\mathrm{III}}$ photocatalyst. The resulting carbocation $\mathbf{D}$ is then trapped by halide anion to produce the final ATRA-type product. An alternative radical-chain propagation cannot be excluded, but is probably not a favored pathway.

Redox potential analysis has been posted to explain the differences between this protocol and Jui's works ${ }^{56,59}$ in mechanism (Fig. 5b, c). In this work, the desired pyridyl radical is generated via an oxidative quenching activation. The protonated halopyridyl trifluoroacetate possessing higher reduction potential $\left(E_{1 / 2}\right.$ red $\left.=-0.69 \mathrm{~V}\right)$ is facial to be reduced by excited state $*[\mathrm{Ir}]^{\mathrm{III}}\left[E_{1 / 2}\left({ }^{*} \mathrm{Ir}{ }^{\mathrm{III}} / \mathrm{Ir}^{\mathrm{IV}}\right)=-0.96 \mathrm{~V}\right]$. After radical addition, redox potential of the alkyl radical $\mathrm{C}\left(E_{1 / 2}{ }^{\mathrm{Oxi}}=0.47 \mathrm{~V}\right)^{70}$ and $[\mathrm{Ir}]^{\mathrm{IV}}\left[E_{1 /}\right.$ $\left.{ }_{2}\left(\mathrm{Ir}^{\mathrm{IV}} / \mathrm{Ir}^{\mathrm{III}}\right)=1.21 \mathrm{~V}\right]$ matches each other, occurring SET to produce carbocation $\mathbf{D}$ and regenerate $[\mathrm{Ir}]^{\mathrm{III}}$. Contrarily, Jui's works utilized the reductive quenching by $\mathrm{HEH}$ to give [Ir] ${ }^{\mathrm{II}}\left[E_{1}\right.$ / $\left.{ }_{2}\left(\mathrm{Ir}^{\mathrm{II}} / \mathrm{Ir}^{\mathrm{III}}\right)=-1.51 \mathrm{~V}\right]$. Then pyridyl radical was generated from the reductive activation of halopyridines $\left(E_{1 / 2}\right.$ red $\left.=-1.1 \mathrm{~V}\right)$. So hantzsch ester (HEH) is indispensable in Jui's conditions for the reductive quenching cycle which is obstacle for the halopyridylation of alkenes (Fig. 5d).

Finally, gram-scale reactions and further transformations of this protocol were conducted to demonstrate the synthetic utility. As shown in Fig. 6, 84\%, 89\%, and 75\% yields of chloro-, bromo-, and iodo-pyridylation products could be isolated respectively under standard conditions (3ma, 3ga, and 3pa). Furthermore, the $\mathrm{C}-\mathrm{X}$ bonds of these products are beneficial for performing further synthetic elaborations. It have been showed that the halopyridination products $3 \mathrm{ga}$ and $\mathbf{3 m a}$ could undergo many useful transformations, such elimination ( $7 \mathbf{a}$ and $7 \mathbf{b})$, and substitution by sulfinate $(7 \mathbf{c})$, thiocyanate $(7 \mathbf{d})$, thiophenol $(7 \mathbf{e})$, and azidation ( $7 \mathbf{f}$ and $\mathbf{7} \mathbf{g}$ ) in moderate to good yields (Fig. 6).

\section{Discussion}

In this work, we have developed a photo-induced catalytic halopyridylation of unactivated alkenes. Using halogenated pyridines as dual pyridyl and halogen source, new $\mathrm{C}-\mathrm{C}$ bond and $\mathrm{C}$ $-\mathrm{X}$ bond are simultaneously and regioselectively constructed under mild conditions. It also features an excellent atom and step economy for the late-stage halopyridylation of complex molecules. Tackling the challenging issue from $\beta$ - $\mathrm{H}$ elimination and alkyl $\mathrm{C}-\mathrm{X}$ cleavage, this photo-induced catalysis complements traditional transition metal catalysis in terms of mechanism and product diversity. Moreover, this protocol has good synthetic utility for scale-up preparation, and the $\mathrm{C}-\mathrm{X}$ bonds of these halopyridination products can serve as versatile handle for further transformations.

\section{Methods}

General procedure for photo-induced catalytic halopyridylation of alkenes. To an oven-dried $4 \mathrm{~mL}$ vial was added $\operatorname{Ir}(\mathrm{ppy})_{2}(\mathrm{dtbbpy}) \mathrm{PF}_{6}(0.005 \mathrm{mmol}, 1.0 \mathrm{~mol} \%)$, halopyridines 1 ( $0.5 \mathrm{mmol}, 1.0$ equiv.), alkenes 2 ( $1.5 \mathrm{mmol}, 3.0$ equiv.), TFA $(0.5 \mathrm{mmol}, 1.0$ equiv.), and TFE $(2.5 \mathrm{~mL}, 0.2 \mathrm{M})$ in the nitrogen glove box. The vial was capped with a septum and wrapped with parafilm. The reaction mixture was stirred for $16 \mathrm{~h}$ under visible light irradiation $\left(1 \times\right.$ Kessil PR160, $\lambda_{\max }=456 \mathrm{~nm}$, $40 \mathrm{~W}$, irradiation temperature maintained between 25 and $30^{\circ} \mathrm{C}$ ). Upon completion, the crude product was neutralized with saturated $\mathrm{NaHCO}_{3}$ solution or $\mathrm{Et}_{3} \mathrm{~N}$ and extracted with ethyl acetate. Organic layer was washed with brine solution and dried over anhydrous $\mathrm{Na}_{2} \mathrm{SO}_{4}$. Removal of the organic solvent in a vacuum rotavapor followed by flash silica gel column chromatographic purification to afford the desired products in moderate to good yields. 


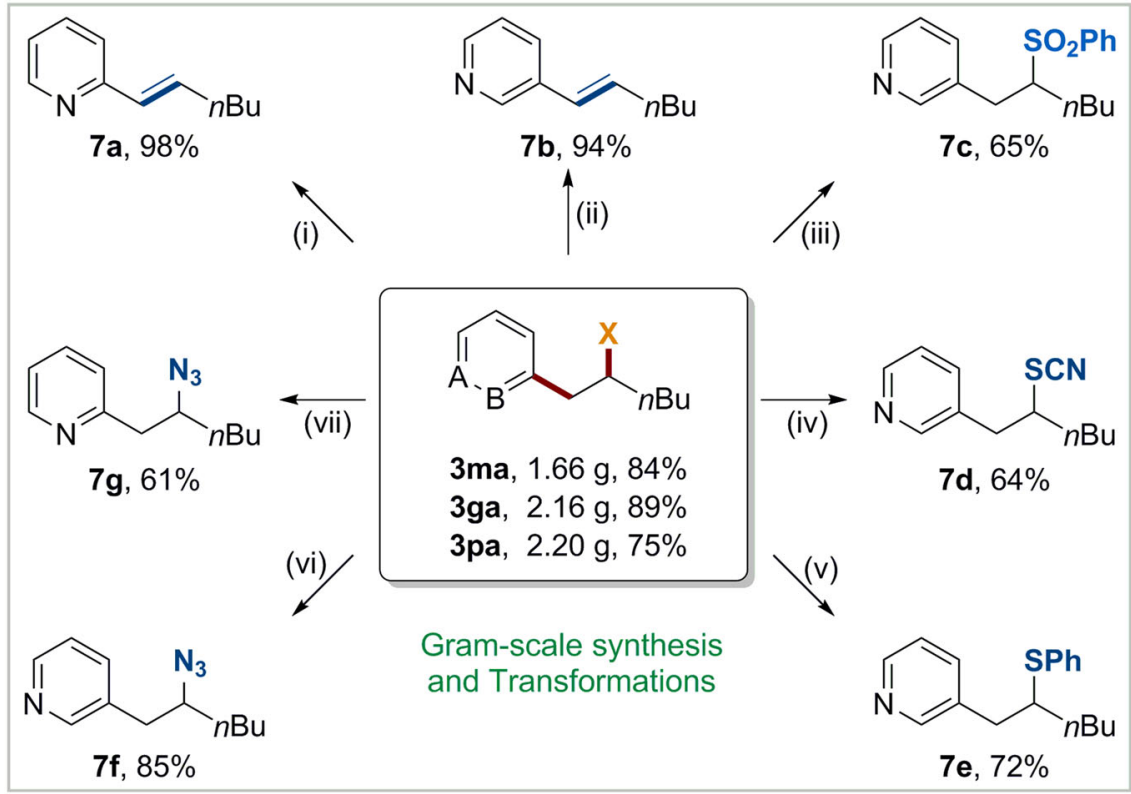

Fig. 6 Synthetic transformations. (i) 3ga, $\mathrm{K}_{2} \mathrm{CO}_{3}, \mathrm{DMF}, 80^{\circ} \mathrm{C}, 6 \mathrm{~h}$. (ii) $3 \mathbf{p a}, \mathrm{K}_{2} \mathrm{CO}_{3}, \mathrm{DMF}, 80^{\circ} \mathrm{C}, 6 \mathrm{~h}$. (iii) $3 \mathrm{pa}, \mathrm{NaSO}{ }_{2} \mathrm{Ph}, \mathrm{DMF}, 60^{\circ} \mathrm{C}, 3 \mathrm{~h}$. (iv) $3 \mathrm{pa}$, $\mathrm{NaSCN}, \mathrm{Na}_{2} \mathrm{CO}_{3}, \mathrm{MeCN}, 40^{\circ} \mathrm{C}, 3 \mathrm{~h}$. (v) 3pa, thiophenol, $\mathrm{Na}_{2} \mathrm{CO}_{3}, \mathrm{MeCN}, 40^{\circ} \mathrm{C}, 7 \mathrm{~h}$. (vi) 3pa, $\mathrm{NaN}_{3}, \mathrm{DMF}, 60^{\circ} \mathrm{C}, 10 \mathrm{~h}$. (vii) 3ga, $\mathrm{NaN}$, $\mathrm{DMF}, 60^{\circ} \mathrm{C}, 10 \mathrm{~h}$.

\section{Data availability}

The authors declare that the data supporting the findings of this study, including experimental details and compound characterization, are available within the article and its Supplementary Information files. All data are available from the corresponding author upon request.

Received: 1 July 2021; Accepted: 18 October 2021;

Published online: 11 November 2021

\section{References}

1. McDonald, R. I., Liu, G. \& Stahl, S. S. Palladium(II)-catalyzed alkene functionalization via nucleopalladation: stereochemical pathways and enantioselective catalytic applications. Chem. Rev. 111, 2981-3019 (2011).

2. Yin, G., Mu, X. \& Liu, G. Palladium(II)-catalyzed oxidative difunctionalization of alkenes: bond forming at a high-valent palladium center. Acc. Chem. Res. 49, 2413-2423 (2016).

3. Cheng, Q. et al. Iridium-catalyzed asymmetric allylic substitution reactions. Chem. Rev. 119, 1855-1969 (2019).

4. Kalyani, D., Satterfield, A. D. \& Sanford, M. S. Palladium-catalyzed oxidative arylhalogenation of alkenes: synthetic scope and mechanistic insights. J. Am. Chem. Soc. 132, 8419-8427 (2010).

5. García-Domínguez, A., Li, Z. \& Nevado, C. Nickel-catalyzed reductive dicarbofunctionalization of alkenes. J. Am. Chem. Soc. 139, 6835-6838 (2017).

6. Legnani, L., Prina-Cerai, G., Delcaillau, T., Willems, S. \& Morandi, B. Efficient access to unprotected primary amines by iron-catalyzed aminochlorination of alkenes. Science 362, 434-438 (2018).

7. Lear, J. M. et al. Multi-component heteroarene couplings via polarity-reversed radical cascades. Chem. Commun. 55, 8820-8823 (2019).

8. Miller, D. C. et al. Anti-Markovnikov hydroamination of unactivated alkenes with primary alkyl amines. J. Am. Chem. Soc. 141, 16590-16594 (2019).

9. Tu, H. Y. et al. Enantioselective three-component fluoroalkylarylation of unactivated olefins through nickel-catalyzed cross-electrophile coupling. J. Am. Chem. Soc. 142, 9604-9611 (2020).

10. Heck, R. F. Palladium-catalyzed reactions of organic halides with olefins. Acc. Chem. Res. 12, 146-151 (1979).

11. Beletskaya, I. P. \& Cheprakov, A. V. The heck reaction as a sharpening stone of palladium catalysis. Chem. Rev. 100, 3009-3066 (2000).

12. Torborg, C. \& Beller, M. Recent applications of palladium-catalyzed coupling reactions in the pharmaceutical, agrochemical, and fine chemical industries. Adv. Syth. Catal. 351, 3027-3043 (2009).

13. Johansson Seechurn, C. C. C., Kitching, M. O., Colacot, T. J. \& Snieckus, V. Palladium-catalyzed cross-coupling: a historical contextual perspective to the 2010 nobel prize. Angew. Chem. Int. Ed. 51, 5062-5085 (2012).
14. Bhakta, S. \& Ghosh, T. Emerging nickel catalysis in heck reactions: recent developments. Adv. Syth. Catal. 362, 5257-5274 (2020)

15. Gao, P. \& Cook, S. P. A reductive-heck approach to the hydroazulene ring system: a formal synthesis of the englerins. Org. Lett. 14, 3340-3343 (2012).

16. Shen, C. et al. Enantioselective arylative dearomatization of indoles via Pdcatalyzed intramolecular reductive heck reactions. J. Am. Chem. Soc. 137, 4936-4939 (2015).

17. Gurak, J. A. \& Engle, K. M. Practical intermolecular hydroarylation of diverse alkenes via reductive heck coupling. ACS Catal. 8, 8987-8992 (2018).

18. Oxtoby, L. J., Gurak, J. A. Jr., Wisniewski, S. R., Eastgate, M. D. \& Engle, K. M. Palladium-catalyzed reductive heck coupling of alkenes. Trends Chem. 1, 572-587 (2019)

19. Murphy, J. M., Liao, X. \& Hartwig, J. F. Meta halogenation of 1,3-disubstituted arenes via iridium-catalyzed arene borylation. J. Am. Chem. Soc. 129, 15434-15435 (2007)

20. Dai, C., Narayanam, J. M. R. \& Stephenson, C. R. J. Visible-light-mediated conversion of alcohols to halides. Nat. Chem. 3, 140-145 (2011).

21. Teskey, C. J., Lui, A. Y. W. \& Greaney, M. F. Ruthenium-catalyzed metaselective C-H bromination. Angew. Chem. Int. Ed. 54, 11677-11680 (2015).

22. Petrone, D. A., Ye, J. \& Lautens, M. Modern transition-metal-catalyzed carbon-halogen bond formation. Chem. Rev. 116, 8003-8104 (2016).

23. Latham, J., Brandenburger, E., Shepherd, S. A., Menon, B. R. K. \& Micklefield, J. Development of halogenase enzymes for use in synthesis. Chem. Rev. 118, 232-269 (2018).

24. Wappes, E. A., Vanitcha, A. \& Nagib, D. A. $\beta$ C-H di-halogenation via iterative hydrogen atom transfer. Chem. Sci. 9, 4500-4504 (2018).

25. Song, S. et al. DMSO-catalysed late-stage chlorination of (hetero)arenes. Nat. Catal. 3, 107-115 (2020).

26. Liu, H., Li, C., Qiu, D. \& Tong, X. Palladium-catalyzed cycloisomerizations of (Z)-1-Iodo-1,6-dienes: iodine atom transfer and mechanistic insight to alkyl iodide reductive elimination. J. Am. Chem. Soc. 133, 6187-6193 (2011).

27. Newman, S. G., Howell, J. K., Nicolaus, N. \& Lautens, M. Palladium-catalyzed carbohalogenation: bromide to iodide exchange and domino processes. J. Am. Chem. Soc. 133, 14916-14919 (2011).

28. Newman, S. G. \& Lautens, M. Palladium-catalyzed carboiodination of alkenes: carbon-carbon bond formation with retention of reactive functionality. J. Am. Chem. Soc. 133, 1778-1780 (2011).

29. Jia, X., Petrone, D. A. \& Lautens, M. A conjunctive carboiodination: indenes by a double carbopalladation-reductive elimination domino process. Angew. Chem. Int. Ed. 51, 9870-9872 (2012).

30. Petrone, D. A., Malik, H. A., Clemenceau, A. \& Lautens, M. Functionalized chromans and isochromans via a diastereoselective $\mathrm{Pd}(0)$-catalyzed carboiodination. Org. Lett. 14, 4806-4809 (2012).

31. Petrone, D. A., Yoon, H., Weinstabl, H. \& Lautens, M. Additive effects in the palladium-catalyzed carboiodination of chiral $\mathrm{N}$-allyl carboxamides. Angew. Chem. Int. Ed. 53, 7908-7912 (2014). 
32. Yoon, H., Marchese, A. D. \& Lautens, M. Carboiodination catalyzed by nickel. J. Am. Chem. Soc. 140, 10950-10954 (2018).

33. Marchese, A. D., Kersting, L. \& Lautens, M. Diastereoselective nickel-catalyzed carboiodination generating six-membered nitrogen-based heterocycles. Org. Lett. 21, 7163-7168 (2019).

34. Marchese, A. D., Lind, F., Mahon, Á. E., Yoon, H. \& Lautens, M. Forming benzylic iodides via a nickel catalyzed diastereoselective dearomative carboiodination reaction of indoles. Angew. Chem. Int. Ed. 58, 5095-5099 (2019).

35. Zhang, Z.-M. et al. Palladium/XuPhos-catalyzed enantioselective carboiodination of olefin-tethered aryl iodides. J. Am. Chem. Soc. 141, 8110-8115 (2019).

36. Pan, H. et al. Photo-induced chloride atom transfer radical addition and aminocarbonylation reactions. Asian J. Org. Chem. 8, 1513-1518 (2019).

37. Narayanam, J. M. R. \& Stephenson, C. R. J. Visible light photoredox catalysis: applications in organic synthesis. Chem. Soc. Rev. 40, 102-113 (2011).

38. Gentry, E. C. \& Knowles, R. R. Synthetic applications of proton-coupled electron transfer. Acc. Chem. Res. 49, 1546-1556 (2016).

39. Hopkinson, M. N., Tlahuext-Aca, A. \& Glorius, F. Merging visible light photoredox and gold catalysis. Acc. Chem. Res. 49, 2261-2272 (2016)

40. Romero, N. A. \& Nicewicz, D. A. Organic photoredox catalysis. Chem. Rev 116, 10075-10166 (2016).

41. Skubi, K. L., Blum, T. R. \& Yoon, T. P. Dual catalysis strategies in photochemical synthesis. Chem. Rev. 116, 10035-10074 (2016).

42. Tellis, J. C. et al. Single-electron transmetalation via photoredox/nickel dual catalysis: unlocking a new paradigm for $\mathrm{sp}(3)-\mathrm{sp}(2)$ cross-coupling. Acc. Chem. Res. 49, 1429-1439 (2016).

43. Hu, A., Guo, J.-J., Pan, H. \& Zuo, Z. Selective functionalization of methane, ethane, and higher alkanes by cerium photocatalysis. Science 361, 668-672 (2018).

44. Capacci, A. G., Malinowski, J. T., McAlpine, N. J., Kuhne, J. \& MacMillan, D. W. C. Direct, enantioselective a-alkylation of aldehydes using simple olefins. Nat. Chem. 9, 1073-1077 (2017).

45. Musacchio, A. J. et al. Catalytic intermolecular hydroaminations of unactivated olefins with secondary alkyl amines. Science 355, 727-730 (2017).

46. Monos, T. M., McAtee, R. C. \& Stephenson, C. R. J. Arylsulfonylacetamides as bifunctional reagents for alkene aminoarylation. Science 361, 1369-1373 (2018).

47. Huang, L. et al. Cascade cross-coupling of dienes: photoredox and nickel dual catalysis. Angew. Chem. Int. Ed. 59, 457-464 (2020).

48. Song, F. et al. Visible-light-enabled stereodivergent synthesis of E- and Z-configured 1,4-dienes by photoredox/nickel dual catalysis. Angew. Chem. Int. Ed. 59, 177-181 (2020).

49. El-Maksouda, S. A. A. \& Fouda, A. S. Some pyridine derivatives as corrosion inhibitors for carbon steel in acidic medium. Mater. Chem. Phys. 93, 84-90 (2005).

50. Roughley, S. D. \& Jordan, A. M. The medicinal chemist's toolbox: an analysis of reactions used in the pursuit of drug candidates. J. Med. Chem. 54, 3451-3479 (2011).

51. Sevov, C. S. et al. Evolutionary design of low molecular weight organic anolyte materials for applications in nonaqueous redox flow batteries. J. Am. Chem. Soc. 137, 14465-14472 (2015).

52. Alvarez, R. et al. Pyridine based antitumour compounds acting at the colchicine site. Curr. Med. Chem. 23, 1100-1130 (2016).

53. Rossler, S. L. et al. Pyridinium salts as redox-active functional group transfer reagents. Angew. Chem. Int. Ed. 59, 9264-9280 (2020).

54. Aycock, R. A., Vogt, D. B. \& Jui, N. T. A practical and scalable system for heteroaryl amino acid synthesis. Chem. Sci. 8, 7998-8003 (2017).

55. Aycock, R. A., Wang, H. \& Jui, N. T. A mild catalytic system for radical conjugate addition of nitrogen heterocycles. Chem. Sci. 8, 3121-3125 (2017).

56. Boyington, A. J., Riu, M.-L. Y. \& Jui, N. T. Anti-markovnikov hydroarylation of unactivated olefins via pyridyl radical intermediates. J. Am. Chem. Soc. 139, 6582-6585 (2017).

57. Chen, D. et al. Metal-free, intermolecular carbopyridylation of alkenes via visible-light-induced reductive radical coupling. Chem. Sci. 9, 9012-9017 (2018).

58. He, Y.-T., Kang, D., Kim, I. \& Hong, S. Metal-free photocatalytic trifluoromethylative pyridylation of unactivated alkenes. Green. Chem. 20 5209-5214 (2018).

59. Seath, C. P., Vogt, D. B., Xu, Z., Boyington, A. J. \& Jui, N. T. Radical hydroarylation of functionalized olefins and mechanistic investigation of photocatalytic pyridyl radical reactions. J. Am. Chem. Soc. 140, 15525-15534 (2018).

60. Boyington, A. J., Seath, C. P., Zearfoss, A. M., Xu, Z. \& Jui, N. T. Catalytic strategy for regioselective arylethylamine synthesis. J. Am. Chem. Soc. 141, 4147-4153 (2019).
61. Chen, J., Zhu, S., Qin, J. \& Chu, L. Intermolecular, redox-neutral azidoarylation of alkenes via photoredox catalysis. Chem. Commun. 55, 2336-2339 (2019)

62. Moon, Y. et al. Visible light induced alkene aminopyridylation using $\mathrm{N}$-aminopyridinium salts as bifunctional reagents. Nat. Commun. 10, 4117 (2019).

63. Mathi, G. R., Jeong, Y., Moon, Y. \& Hong, S. Photochemical carbopyridylation of alkenes using $\mathrm{N}$-alkenoxypyridinium salts as bifunctional reagents. Angew. Chem. Int. Ed. 59, 2049-2054 (2020).

64. Hu, Y.-C., Ji, D.-W., Zhao, C.-Y., Zheng, H. \& Chen, Q.-A. Catalytic prenylation and reverse prenylation of indoles with isoprene: regioselectivity manipulation through choice of metal hydride. Angew. Chem. Int. Ed. 58, 5438-5442 (2019)

65. Ji, D.-W. et al. A regioselectivity switch in Pd-catalyzed hydroallylation of alkynes. Chem. Sci. 10, 6311-6315 (2019).

66. Kuai, C.-S. et al. Ligand-regulated regiodivergent hydrosilylation of isoprene under iron catalysis. Angew. Chem. Int. Ed. 59, 19115-19120 (2020).

67. Min, X. T. et al. Visible light induced bifunctional rhodium catalysis for decarbonylative coupling of imides with alkynes. Angew. Chem. Int. Ed. 60, 1583-1587 (2021).

68. Wallentin, C.-J., Nguyen, J. D., Finkbeiner, P. \& Stephenson, C. R. J. Visible light-mediated atom transfer radical addition via oxidative and reductive quenching of photocatalysts. J. Am. Chem. Soc. 134, 8875-8884 (2012).

69. Tomita, R., Yasu, Y., Koike, T. \& Akita, M. Combining photoredox-catalyzed trifluoromethylation and oxidation with DMSO: facile synthesis of $\alpha$ trifluoromethylated ketones from aromatic alkenes. Angew. Chem. Int. Ed. 53, 7144-7148 (2014)

70. Wayner, D. D. M. \& Houmam, A. Redox properties of free radicals. Acta Chem. Scand. 52, 377-384 (1998).

\section{Acknowledgements}

We thank Prof. Daniel K. Kim (Temple University) and Prof. Zhi-Shi Ye (DUT) for helpful discussions and manuscript revisions. Financial support from the National Natural Science Foundation of China (22071239), Dalian Institute of Chemical Physics (DICPI201902), and Doctoral Research Startup Fund Project of Liaoning Province (2020BS017) is acknowledged.

\section{Author contributions}

Q.-A.C. conceived and supervised the project. Q.-A.C. and S.-Y.G. designed the experiments. S.-Y.G., F.Y., T.-T.S., Y.-Q.G., X.-T.M., D.-W.J., and Y.-C. H. performed the experiments and analyzed the data. All authors discussed the results and commented on the manuscript.

\section{Competing interests}

The authors declare no competing interests.

\section{Additional information}

Supplementary information The online version contains supplementary material available at https://doi.org/10.1038/s41467-021-26857-w.

Correspondence and requests for materials should be addressed to Qing-An Chen.

Peer review information Nature Communications thanks Aaron Vannucci and the anonymous reviewer(s) for their contribution to the peer review of this work.

Reprints and permission information is available at http://www.nature.com/reprints

Publisher's note Springer Nature remains neutral with regard to jurisdictional claims in published maps and institutional affiliations.

\section{(i)}

Open Access This article is licensed under a Creative Commons Attribution 4.0 International License, which permits use, sharing, adaptation, distribution and reproduction in any medium or format, as long as you give appropriate credit to the original author(s) and the source, provide a link to the Creative Commons license, and indicate if changes were made. The images or other third party material in this article are included in the article's Creative Commons license, unless indicated otherwise in a credit line to the material. If material is not included in the article's Creative Commons license and your intended use is not permitted by statutory regulation or exceeds the permitted use, you will need to obtain permission directly from the copyright holder. To view a copy of this license, visit http://creativecommons.org/ licenses/by/4.0/.

(C) The Author(s) 2021 